\title{
Locomotor Training Maintains Normal Inhibitory Influence on Both Alpha- and Gamma-Motoneurons after Neonatal Spinal Cord Transection
}

\author{
Ronaldo M. Ichiyama, ${ }^{1,2}$ Jonas Broman, ${ }^{6}$ Roland R. Roy, ${ }^{5}$ Hui Zhong, ${ }^{2}$ V. Reggie Edgerton, ${ }^{2,3,5}$ and Leif A. Havton ${ }^{4,5}$ \\ ${ }^{1}$ Institute of Membrane and Systems Biology, University of Leeds, Leeds LS2 9JT, United Kingdom, Departments of ${ }^{2}$ Physiological Science, ${ }^{3}$ Neurobiology, \\ and ${ }^{4} \mathrm{Neurology}$, and ${ }^{5} \mathrm{Brain}$ Research Institute, University of California, Los Angeles, California 90095, and ${ }^{6}$ Department of Neuroscience, Karolinska \\ Institute, S-171 77 Stockholm, Sweden
}

Spinal cord injuries lead to impairments, which are accompanied by extensive reorganization of neuronal circuits caudal to the injury. Locomotor training can aid in the functional recovery after injury, but the neuronal mechanisms associated with such plasticity are only sparsely known. We investigated ultrastructurally the synaptic inputs to tibialis anterior motoneurons (MNs) retrogradely labeled in adult rats that had received a complete midthoracic spinal cord transection at postnatal day 5. A subset of the injured rats received locomotor training. Both $\gamma$ - and $\alpha$-MNs were studied. The total number of boutons apposing $\gamma$-MNs, but not $\alpha$-MNs, was reduced after neonatal spinal cord transection. The proportion of inhibitory to excitatory boutons, however, was increased significantly in both $\alpha$-MNs and $\gamma$-MNs in spinally transected rats, but with locomotor training returned to levels observed in intact rats. The specific densities and compositions of synaptic boutons were, however, different between all three groups. Surprisingly, we observed the atypical presence of both C-and M-type boutons apposing the somata of $\gamma$-MNs in the spinal rats, regardless of training status. We conclude that a neonatal spinal cord transection induces significant reorganization of synaptic inputs to spinal motoneurons caudal to the site of injury with a net increase in inhibitory influence, which is associated with poor stepping. Spinal cord injury followed by successful locomotor training, however, results in improved bipedal stepping and further synaptic changes with the proportion of inhibitory and excitatory inputs to the motoneurons being similar to that observed in intact rats.

\section{Introduction}

Spinal cord injuries (SCI) result in a combination of motor, sensory, and autonomic impairments. Interestingly, extensive neurochemical, electrophysiological, and structural plasticity of the neuronal circuitry can take place spontaneously in spinal cord segments caudal to the injury (Raineteau and Schwab, 2001; Wolpaw and Tennissen, 2001; Tillakaratne et al., 2002; Bareyre et al., 2004; Edgerton et al., 2004; Frigon and Rossignol, 2006). Activity-induced plasticity also can be induced within the segments below the level of an SCI in both animal models and humans (Edgerton et al., 2004; Dietz, 2009). For instance, locomotor training after a complete spinal cord transection (ST) in adult cats results in partial recovery of gait and an amelioration of lesion-induced augmentation of inhibitory influences within the ventral horn (de Leon et al., 1998; Tillakaratne et al., 2002). Similarly, step training in rats with an ST at

Received Dec. 30, 2009; revised Sept. 6, 2010; accepted Sept. 13, 2010.

This work was supported by National Institutes of Health Grants NS 16333 and NS 40917, Christopher and Dana Reeve Foundation (Grant VEC 2002), State of California Roman Reed Funds, Dr. Miriam and Sheldon G. Adelson Medical Research Foundation, and the Swedish Research Council (Project Number 14276). We thank Birgitta Sjöstrand for excellent technical support of the ultrastructural studies, Maynor Herrera for expert animal care and surgical support, and all the tireless volunteer undergraduate students for caring for and training all rats.

Correspondence should be addressed to Dr. Leif A. Havton, Department of Neurology, David Geffen School of Medicine at UCLA, Neuroscience Research Building, 635 Charles E. Young Drive South, Los Angeles, CA 90095. E-mail: Ihavton@mednet.ucla.edu.

DOI:10.1523/JNEUROSCI.6433-09.2011

Copyright $\odot 2011$ the authors $\quad 0270-6474 / 11 / 310026-08 \$ 15.00 / 0$ a neonatal stage results in pronounced changes in the functional properties of the spinal cord circuitry, including a training-induced increase in the synaptic activation of motoneurons by primary afferents or intramedullary white matter tract stimulation (Petruska et al., 2007). The mechanisms underlying spontaneous and activityinduced plasticity of the neuronal circuitry caudal to a neonatal SCI, however, are not well understood.

The purpose of the present study was to determine the level and type of synaptic reorganization that occurs on motoneurons (MN) after a complete midthoracic spinal cord transection at a neonatal age. We also examined whether step training would affect this reorganization. Quantitative electron microscopic analysis of synaptic inputs onto retrogradely labeled spinal MNs was performed after a complete spinal cord transection in neonatal rats and after daily locomotor training. Our previously reported ultrastructural data on synaptic inputs to MNs in neurologically intact rats were used for control purposes (Ichiyama et al., 2006). We demonstrate a pronounced injury-induced increase in the ratio of inhibitory to excitatory synapses in apposition with both $\alpha$-MNs and $\gamma$-MNs, but this ratio returns to normal in rats that successfully recover stepping in response to locomotor training.

\section{Materials and Methods}

Three groups of rats were studied: ST at postnatal day 5 (P5) and not trained (ST-Non-Tr, $n=3$ ), ST plus step trained (ST-Tr, $n=4)$, and data from intact rats $(n=3)$ from our recent publication (Ichiyama et al., 
2006). All surgical procedures were performed under aseptic conditions. All animal procedures followed the guidelines of the NIH Guide for the Care and Use of Laboratory Animals and were approved by the Chancellor's Animal Research Committee at University of California Los Angeles.

\section{Surgical procedures}

Spinal cord transections in neonatal rats were performed according to established protocols (Kubasak et al., 2005). Briefly, P5 Sprague Dawley female pups were anesthetized deeply via hypothermia. A partial laminectomy was performed between spinal segments T6 and T8 and the spinal cord was completely transected with microscissors. The cut ends of the spinal cord were lifted with small forceps to assure the completeness of the transection and gelfoam was placed into the tissue gap. The muscles and skin were subsequently sutured in layers. Pups were kept with their mothers until P21 when they were weaned and individually housed for the remainder of the postoperative period in a room with a 12:12 $\mathrm{h}$ light/dark cycle with food and water access ad libitum.

\section{Locomotor training and behavioral testing}

Locomotor training started immediately after weaning. Rats of both experimental groups began the experiment with the same neurological deficits. When training was started, the neonatal ST-rats could step with weight support. They also were able to use their limbs for weight support while in their cages. As their body weight increased over time, however, they were not able to maintain independent weight support. For locomotor training, each ST-Tr rat was placed in an upper body harness that was attached to a servo-controlled body weight support system as previously described in detail (Timoszyk et al., 2005). Upper body support was provided such that the rats assumed a bipedal posture, i.e., standing on their hindlimbs. Rats were trained to step in this bipedal posture at varying treadmill speeds. Initially, treadmill belt speed was set at $6 \mathrm{~cm} / \mathrm{s}$, gradually increased to $13.5 \mathrm{~cm} / \mathrm{s}$, and finally to $21 \mathrm{~cm} / \mathrm{s}$. By the end of the training period all rats were stepping at $21 \mathrm{~cm} / \mathrm{s}$ for $20 \mathrm{~min}$. Locomotor training was performed $5 \mathrm{~d}$ a week for 6 weeks.

Step kinematics of each rat were analyzed using a video-based system (Peak Motus, Vicon) that allowed for three-dimensional reconstruction of locomotor behaviors. This test was performed at the end of the training period $(\sim$ P 56$)$ and histological analyses were performed after the final test. The right and left iliac crests, greater trochanters, lateral condyles, lateral malleoli, and the distal end of the fifth metatarsals were labeled with retro-reflective markers. Stepping performance at 6, 13.5, and $21 \mathrm{~cm} / \mathrm{s}$ were recorded using four cameras at a rate of $60 \mathrm{~Hz}$. Data were collected from a continuous bout of $10 \mathrm{~s}$ at each treadmill speed and statistical comparisons were performed using the average scores for individual rats for each condition.

\section{Electron microscopy}

Retrograde labeling of motoneurons. Retrograde labeling of motoneurons and subsequent preparation of spinal cord tissues were performed immediately after termination of the training period for the ST-Tr rats and after the same amount of time for the ST-Non-Tr (i.e., 5 weeks after weaning) and according to our established protocols (Ichiyama et al., 2006). Briefly, under general isoflurane anesthesia, the tibialis anterior (TA) muscle was exposed bilaterally by skin incisions over the anterior aspect of the legs. Horseradish peroxide (HRP, type VI; Sigma; $25 \%$ solution in sterile water) was injected slowly into the muscle (total $4 \mu \mathrm{l}$, injected at four sites with $1 \mu \mathrm{l}$ each) using a Hamilton syringe. Following each injection, the needle was held in place for several seconds to reduce the risk of tracer contamination of adjacent tissues and cotton pellets were used to absorb any leakage at the injection site. The site also was rinsed with a saline solution. The overlying skin was closed using 4.0 Ethilon sutures, and the animals were allowed to fully recover in an incubator before being returned to their home cage.

Tissue preparation. All rats were perfused intracardially at four $\mathrm{d}$ after HRP injection into the TA muscles. Under deep anesthesia (sodium pentobarbital, $100 \mathrm{mg} / \mathrm{kg}$, i.p.), the rats received a brief intravascular rinse with PBS ( $50 \mathrm{cc}$ total volume) and were then perfused with a fixative (1\% paraformaldehyde and $2 \%$ glutaraldehyde in PBS, $\mathrm{pH} 7.4$ ) for 30 $\min$ at $4^{\circ} \mathrm{C}$. To remove excess fixative and terminate the perfusion, a second brief intravascular rinse with PBS was performed. The lumbosacral spinal cord then was removed and placed in PBS at $4^{\circ} \mathrm{C}$.

Histochemistry for HRP detection. Serial transverse sections $(40 \mu \mathrm{m})$ of spinal segments L4-L5 were cut using an oscillating tissue slicer (Electron Microscopy Sciences). A modification of Mesulam's protocol (Mesulam, 1978; Mesulam et al., 1980) was used for the HRP reaction with $3,3^{\prime}, 5,5^{\prime}$-tetramethyl benzidine (TMB) as a chromogen, and we substituted ammonium heptamolybdate for sodium ferrocyanide (Olucha et al., 1985). This procedure allows for a light-insensitive reaction at nearneutral $\mathrm{pH}$. Under the light microscope, wet sections containing labeled motoneurons with abundant TMB reaction product were selected for electron microscopy (EM) processing.

Tissue processing for EM. Sections containing labeled motoneurons were osmicated at room temperature for $18 \mathrm{~h}$ at pH 5.5 (Henry et al., 1985; Ralston, 1990), rinsed in PBS, dehydrated in graded ethanol, and embedded in Durcupan ACM (Electron Microscopy Sciences). Serial ultrathin sections $(60-70 \mathrm{~nm})$ were cut using a Reichert Ultracut Ultramicrotome, collected on formvar-coated copper one-hole grids, and counterstained with uranyl acetate and lead citrate.

Tissue analysis. Ultrathin sections containing motoneurons clearly labeled with HRP crystals were selected for analysis. Using a JEOL 100 CX transmission electron microscope, all labeled motoneurons demonstrating a nucleus were photographed at a primary magnification of $1900 \times$ and digitally analyzed. Quantitative analyses were performed on single ultrastructural sections on motoneurons that fulfilled all of the criteria mentioned above. The soma measurements were determined using C-Imaging software (Compix). The longest soma diameter $(A)$ and the longest diameter perpendicular to $A(B)$ were used to calculate the mean soma diameter and the soma volume using the formula for an oblate spheroid rotated around the minor transverse axis, i.e., $(\pi / 6) A B^{2}$ (Ulfhake, 1984).

For classification and quantification of bouton measurements, serial electron micrographs of the entire soma cell membrane of labeled TA motoneurons were analyzed at a primary magnification of $7200 \times$. The electron micrographs were combined in composites using Adobe Photoshop 6.0 (Adobe Systems) and manually adjusted for contrast and brightness for optimal visualization of ultrastructural features.

The well established classification of bouton types in apposition to motoneurons was used (Conradi et al., 1979; Brännström, 1993; Novikov et al., 2000; Ichiyama et al., 2006). Briefly, S-type boutons were small in diameter and demonstrated spherical synaptic vesicles and asymmetric postsynaptic densities. F-type boutons also were small in diameter, but exhibited flattened or pleomorphic synaptic vesicles and symmetric postsynaptic densities. C-type boutons were large in diameter and demonstrated one or more subsynaptic cisterns in the postsynaptic element. M-type boutons had the largest diameters, exhibited multiple active zones, and were associated with a characteristic presynaptic P-bouton.

The outline of each individual bouton was traced and bouton area and diameter were calculated using C-imaging (SimplePCI; Hamamatsu). We calculated both the percentage of coverage (percentage of membrane coverage by synaptic boutons) and synaptic density (number of boutons per $100 \mu \mathrm{m}$ ) for all boutons and for individual bouton types. Although the two measures are related, they are both included in the data presentation, as the apposition length normally differs between some bouton types and larger boutons make a relatively bigger contribution to the percentage membrane coverage. The bouton apposition length was measured and used to calculate the percentage synaptic coverage as follows: [(sum of bouton apposition lengths) $\times 100] /[$ (total soma membrane perimeter $)$ - (dendritic holes)].

Statistical analysis. All comparisons between variables involving large and small motoneurons were performed using the nonparametric MannWhitney $U$ test for differences in medians. The only exceptions were the test of differences in bouton types, which was performed using a two-way ANOVA on ranks (Kruskal-Wallis) and the Bonferroni $t$ test for post hoc pairwise multiple comparisons. When not explicitly reported, the significance level was set at $p<0.05$. The statistical comparisons were performed among the three groups using individual $\mathrm{MNs}$ as single observations $(n)$, except for kinematics analyses of the number of steps, where the average for each rat was computed as a single observation $(n$; 
see Locomotor training and behavioral testing, above). Data for individual synaptic apposition lengths and numbers of synaptic boutons were averaged for each $\mathrm{MN}$, and statistical comparisons were performed using MNs as single observations $(n)$ as stated above. Data are reported as mean \pm SEM.

\section{Results}

We performed quantitative ultrastructural studies of the synaptic inputs to 33 TA MNs after a neonatal ST in three ST-Non-Tr rats and to 36 TA MNs after a neonatal ST in four ST-Tr rats. Previously published ultrastructural data on the synaptic inputs of 32 TA MNs in neurologically intact rats served as control data (Ichiyama et al., 2006).

MN soma size is not affected by a neonatal spinal cord transection and locomotor training

A bimodal MN soma size distribution was observed in all groups with soma diameter peaks at $20-25 \mu \mathrm{m}$ and $40-45 \mu \mathrm{m}$ and a trough at $30 \mu \mathrm{m}$ (Fig. $1 \mathrm{~A}$ ). The large (mean soma diameter $>35$ $\mu \mathrm{m}$ ) and small (mean soma diameter $<25 \mu \mathrm{m}$ ) MNs were analyzed separately and are here referred to as $\alpha$ - and $\gamma$-MNs, respectively, based on previously established size criteria (Swett et al., 1986; Destombes et al., 1992; Ichiyama et al., 2006). There were no significant differences in the mean diameter of $\alpha$ - or $\gamma$-MNs after a neonatal ST with $(n=19 \alpha$-MNs and $n=8 \gamma$-MNs $)$ or without ( $n=12 \alpha$-MNs and $n=15 \gamma$-MNs) subsequent locomotor training (Fig. $1 B$ ). These observations are consistent with previous reports (Roy et al., 2007). As in our previous study of TA $\mathrm{MN}$ in intact adult rats (Ichiyama et al., 2006), MNs of an intermediate size (mean soma diameter $>25 \mu \mathrm{m}$ and $<35 \mu \mathrm{m}$ ) were excluded from further quantitative comparisons between groups. This population of MNs was likely to include both $\alpha$ - and $\gamma$-MNs, and possibly $\beta$-MNs (Laporte, 1979).

\section{Synaptic frequency and covering of MNs is affected by ST and} locomotor training

Quantitative analyses of synaptic inputs to TA MNs showed that the percentage membrane coverage of $\gamma$-MNs was significantly lower in ST-Non- $\operatorname{Tr}(n=15 ; 39.5 \pm 5.6 \% ; p<0.05)$ and ST-Tr $(n=8 ; 45.7 \pm 7.2 \% ; p<0.05)$ rats compared with intact rats $(n=10 ; 63.5 \pm 7.3 \%$ ) (Fig. $1 C)$. In contrast, there was no difference in the density of boutons apposing $\alpha$-MNs in intact ( $n=17$; $72.2 \pm 1.7 \%)$, ST-Non- $\operatorname{Tr}(n=12 ; 75.8 \pm 2.3 \%)$, or ST- $\operatorname{Tr}(n=$ $19 ; 76.8 \pm 2.3 \%$ ) rats (Fig. $1 C$ ). Similarly, the number of boutons per $100 \mu \mathrm{m}$ of membrane length was significantly decreased for $\gamma$-MNs in ST-Non- $\operatorname{Tr}(20.4 \pm 2.7)$ and ST-Tr $(24.1 \pm 4.3)$ rats compared with intact rats $(38.7 \pm 4.8)($ Fig. $1 D)$. In contrast, the number of boutons per $100 \mu \mathrm{m}$ of membrane length was unchanged in $\alpha$-MNs $(38.5 \pm 0.9$ intact, $37.7 \pm 2.9$ ST-Non-Tr, $35.1 \pm 1.2 \mathrm{ST}-\mathrm{Tr})($ Fig. $1 \mathrm{D})$.

Inhibitory influence on MNs is elevated after a neonatal ST and restored to intact levels by locomotor training

All boutons apposing the somata of MNs were assigned into one of the four conventional synaptic types: excitatory S-type, inhibitory F-type, large cholinergic C-type, or M-type of primary afferent origin (Conradi et al., 1979; Brännström, 1993; Novikov et al., 2000; Ichiyama et al., 2006) (Fig. 2A-D). Inhibitory influence was assessed as the calculated ratio of F- to S-type boutons (F/S) in apposition with the MN soma membrane for both percentage of membrane coverage and number of boutons per $100 \mu \mathrm{m}$ of membrane length. Four of the $15 \gamma$-MNs in the ST rats were not apposed by any S-type boutons and were excluded from further $\mathrm{F} / \mathrm{S}$ ratio analysis. The F/S ratio for percentage coverage was significantly higher in the ST-Non-Tr rats for both $\gamma$-MNs (7.2 \pm
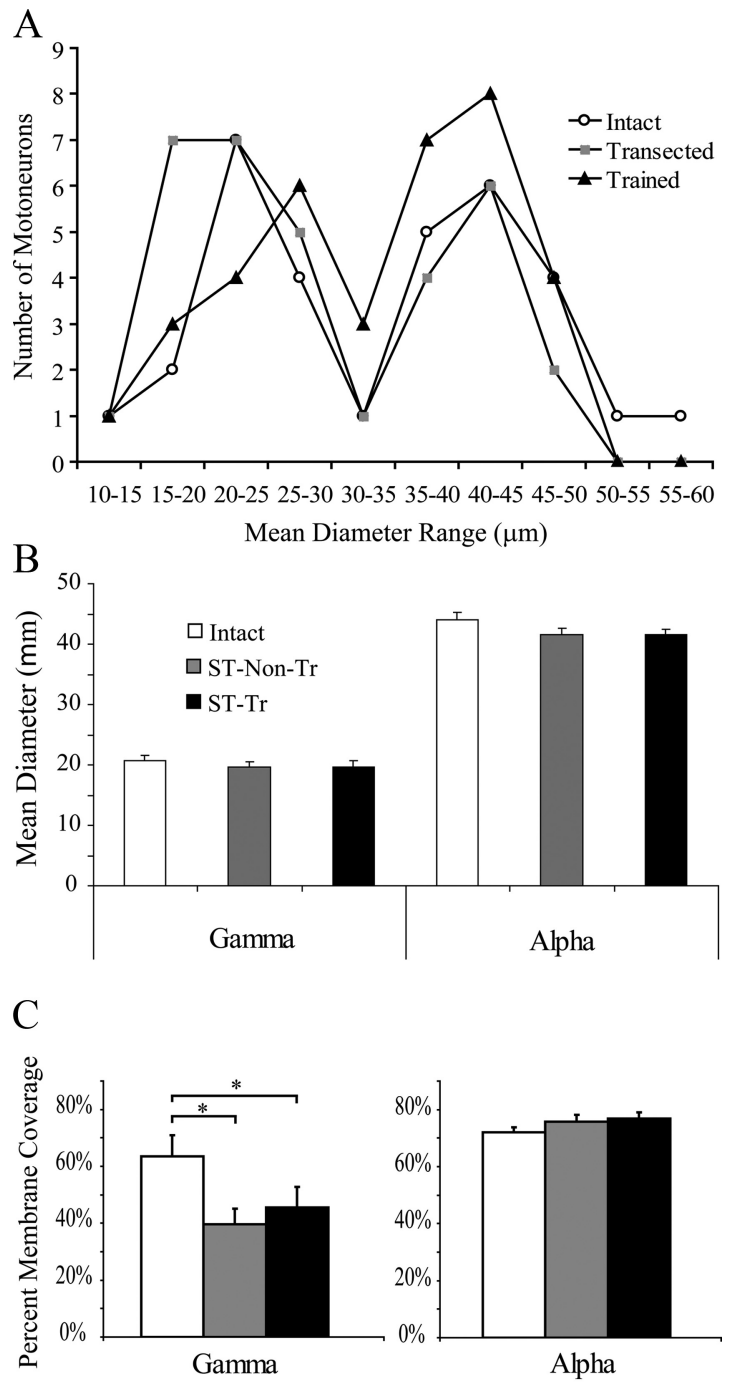

$\mathrm{D}$

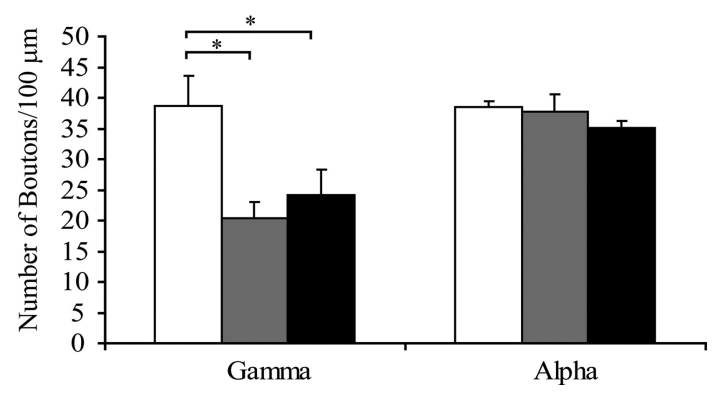

Figure 1. Distribution of all MNs based on mean diameter $(\mu \mathrm{m})$, including intermediary diameter range, which was excluded from synaptology analysis. $A$, Note the bimodal distribution with a clear trough at the $30-35 \mu \mathrm{m}$ range for all groups. $\boldsymbol{B}$, There were no significant differences in the mean diameter of $\gamma$ - or $\alpha$-MNs between the three groups. $\boldsymbol{C}, \boldsymbol{D}$, The total soma membrane synaptic coverage and number of boutons significantly decreased for $\gamma$-MNs after transection injury with and without step training, but were unchanged between all groups for $\alpha$-MNs. ${ }^{*} p<0.05$.

$2.3 ; n=11 ; p<0.005)$ and $\alpha$-MNs $(5.0 \pm 0.3 ; n=12, p<0.001)$ compared with intact rats $(1.7 \pm 0.3, n=10 \gamma$-MNs; $3.7 \pm 0.4$, $n=17 \alpha$-MNs). Interestingly, locomotor training restored the normal balance between inhibitory and excitatory synaptic inputs to both $\alpha$-and $\gamma$-MNs, as the F/S ratios for ST-Tr rats were significantly lower for both $\gamma$-MNs $(1.9 \pm 0.3 ; n=8$; $p<0.005)$ 


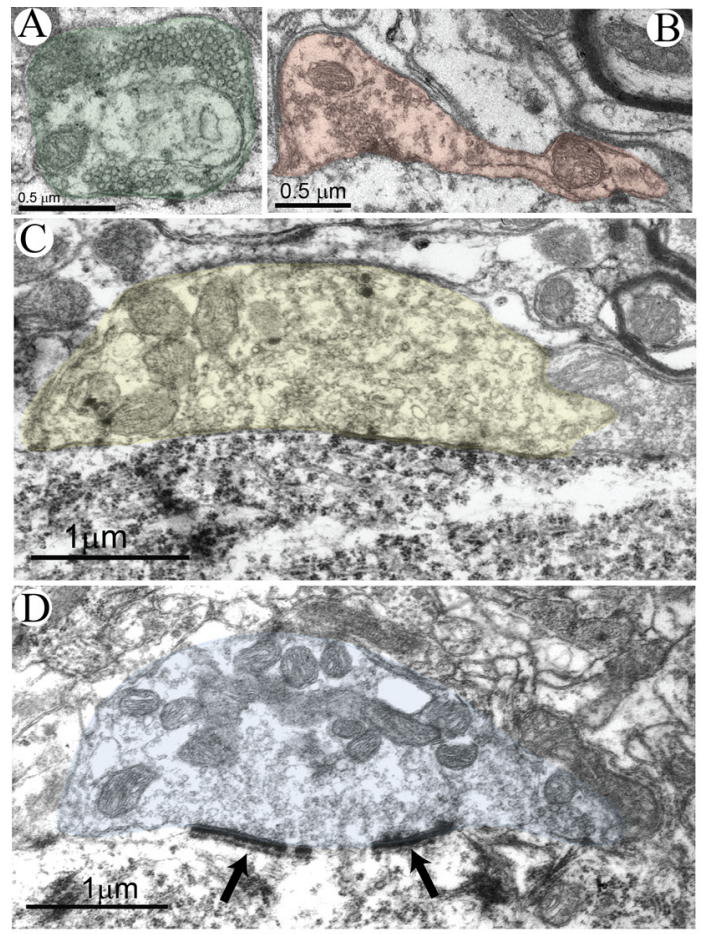

E
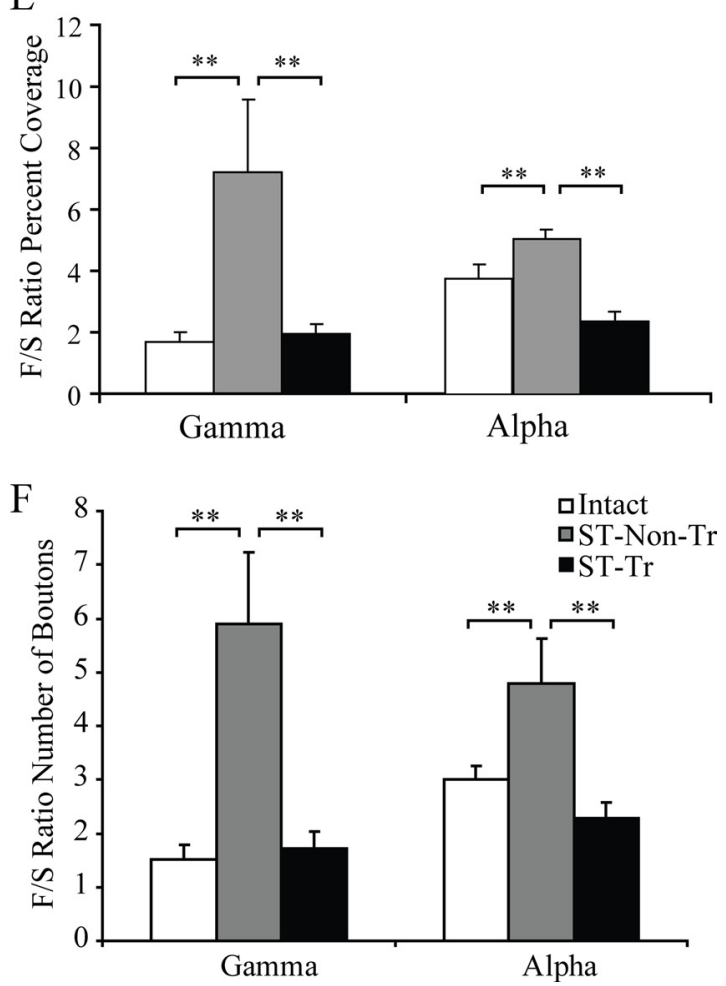

Figure 2. Bouton types in apposition with $\alpha$ - and $\gamma$-MNs in neonatally transected rats. $\boldsymbol{A}-\boldsymbol{D}$, Excitatory $\boldsymbol{S}$-type $(\boldsymbol{A}$; green), inhibitory F-type ( $\boldsymbol{B}$; red), large cholinergic $\mathbf{C}$-type ( $\boldsymbol{C}$; yellow), large M-type ( $\boldsymbol{D}$; blue) with associated Taxi-bodies (arrows). $\boldsymbol{E}, \boldsymbol{F}, \mathrm{F} / \mathrm{S}$ ratios for percentage coverage and number of boutons for $\gamma$ - and $\alpha$-MNs. ${ }^{* *} p<0.005$.

and $\alpha$-MNs $(2.4 \pm 0.3 ; n=19, p<0.001)$ compared with STNon-Tr rats and did not differ significantly from the corresponding ratios in intact rats $(1.7 \pm 0.3, n=10 \gamma$-MNs; $3.7 \pm 0.4, n=$ $17 \alpha$-MNs) (Fig. $2 E$ ). The F/S ratios of the number of boutons per $100 \mu \mathrm{m}$ also were significantly higher for both $\gamma$ - and $\alpha$-MNs in
ST Non-Tr compared with intact rats. These values, however, were similar in intact and ST-Tr rats (Fig. $2 F$ ).

\section{Excitatory and inhibitory inputs to MNs are differentially affected} by ST and locomotor training

The increased inhibitory influence seen in $\gamma$-MNs after ST resulted from the combined effect of a markedly lower membrane coverage by excitatory S-type boutons and fewer S-type boutons per $100 \mu \mathrm{m}$ membrane length in ST-Non-Tr rats $(7.4 \pm 2.3 \%$, $4.01 \pm 1.3$, respectively; $n=15 ; p<0.05)$ compared with intact rats $(25.2 \pm 3.4 \% ; 16.2 \pm 2.1$, respectively; $n=12)$. Concurrently, there was relatively less reduction in the coverage by inhibitory F-type boutons $(28.6 \pm 4.9 \%)$ and in number of F-type boutons per $100 \mu \mathrm{m}$ for ST-Non- $\operatorname{Tr}(16.0 \pm 1.9 ; n=15)$ compared with intact rats $(38.3 \pm 4.9 \% ; 22.5 \pm 3.2$ respectively; $n=$ $12 ; p<0.05$ ) (Fig. $3 A, B$ ). Locomotor training tended to normalize the $\mathrm{F} / \mathrm{S}$ ratio in $\gamma$-MNs by a partial restoration of the excitatory inputs, as the coverage by S-type boutons $(15.5 \pm 3.2 \% ; n=8)$ in ST-Tr rats was not significantly different from that in corresponding intact rats, whereas the number of S-type boutons remained significantly lower in the ST-Tr rats $(9.1 \pm 2.1 ; n=8)$ compared with the ST-Non- $\operatorname{Tr}(n=15)$ and intact rats $(n=12)$. The coverage by F-type boutons $(28.8 \pm 4.6 \% ; p<0.05)$ and the number of F-type boutons (14.3 \pm 2.5$)$, however, remained significantly lower for $\gamma$-MNs in ST-Tr rats.

Although a significant increase in the F/S ratio was observed for $\alpha$-MNs after ST, there were no detectable changes in the membrane coverage by F- and S-type boutons between intact $(n=17)$ and ST-Non-Tr rats $(n=12)$ (Fig. 3C). $\alpha$-MNs, however, showed a relatively modest, but significant, reduction in the number of S-type boutons in ST-Non-Tr rats $(7.1 \pm 1.4 ; n=12)$ compared with intact rats $(9.5 \pm 0.6 ; n=17)$. Both the coverage $(20.1 \pm 1.0 \%)$ and the number $(11.1 \pm 0.9)$ of S-type boutons apposing $\alpha$-MNs, however, were higher in ST-Tr rats $(n=19)$ compared with the intact $(14.5 \pm 1.0 \% ; 9.5 \pm 0.6 ; p<0.005)$ and ST-Non-Tr rats $(10.7 \pm 0.6 \% ; 7.1, \pm 1.4 ; p<0.001)$. ST-Tr rats $(n=19)$ had a significantly smaller percentage $(42.3 \pm 2.4 \%)$ and number (20.6 \pm 1.1$)$ of F-type boutons apposing $\alpha$-MN soma membrane compared with intact $(48.6 \pm 2.0 \% ; 26.4 \pm 0.9 ; n=$ $17 ; p<0.05)$ and ST-Non- $\operatorname{Tr}$ rats $(52.0 \pm 2.2 \% ; 26.9 \pm 1.7 ; n=$ $12 ; p<0.05)$. Thus, the normalization of the $\mathrm{F} / \mathrm{S}$ ratio in $\alpha$-MNs by locomotor training was the net effect of an increase in S-type and a decrease in F-type inputs.

Synaptic inputs by $M$ - and C-type boutons to MNs are affected by a neonatal spinal cord transection and by locomotor training The soma membrane of $\gamma$-MNs is normally devoid of both Cand M-type boutons in neurologically intact rats (Ichiyama et al., 2006). Interestingly, in both ST-Non-Tr and ST-Tr groups, the soma membrane of $\gamma$-MNs was, on occasion, apposed by $\mathrm{C}$ - and M-type boutons (Fig. 4). In $\gamma$-MNs of the ST-Non-Tr rats, C-type boutons apposed 1.2\% and M-type boutons apposed $2.9 \pm 2.0 \%$ of the soma membrane, whereas C-type boutons apposed $1.1 \pm 0.8 \%$ and M-type boutons apposed $1.2 \%$ of the soma membrane of $\gamma$-MNs in the ST-Tr group (Fig. 3A). As only one of $15 \gamma$-MNs in ST-Non-Tr rats demonstrated C-type boutons apposing its soma membrane and only one of eight $\gamma$-MNs demonstrated M-type boutons in the ST-Tr group, no SEM was calculated for those independent variables. In contrast, the soma membrane of $\alpha$-MNs was apposed by both C- and M-type boutons in all groups, but there were no significant differences among the groups for either bouton type. 
Locomotor training results in a behavioral recovery of stepping function

We previously have described a return of treadmill stepping ability in response to daily locomotor training after a neonatal complete spinal cord transection (Petruska et al., 2007). In the present study, we included cage and littermates of the rats used for the above behavioral investigation (Petruska et al., 2007) and the locomotor training was identical for both studies. The rats that were trained daily to step bipedally ( $n=4$, ST-Tr) on a moving treadmill belt (Fig. 5A) performed a larger number of plantar alternating steps than ST-Non-Tr rats $(n=3)$ in a 10 s testing period ( $12 \pm 1.3$ vs $6 \pm 2.6$, respectively) (Fig. $5 B$ ). In addition, the quality of stepping was higher in ST-Tr rats compared with ST-Non-Tr rats, with the trained animals showing more consistent left-right coordination (Fig. 5C,D) and interjoint coordination patterns, as illustrated by the relative motion plots between the ankle and knee joints (Fig. $5 E, F)$. Thus, the behavioral data support the notion that the observed training-induced ultrastructural adaptations in the synaptic inputs to both $\gamma$ - and $\alpha$-MNs after neonatal spinal cord transection is associated with improved stepping function.

\section{Discussion}

We demonstrate that synaptic inputs to retrogradely labeled TA MNs undergo extensive plasticity in adult rats after a neonatal (P5) midthoracic spinal cord complete transection and daily locomotor training. Specifically, ST resulted in an increased inhibitory influence on both $\alpha$ - and $\gamma$-MNs, i.e., a higher proportion of inhibitory F-type boutons compared with excitatory S-type boutons (F/S ratio). Interestingly, baseline levels of inhibitory-toexcitatory bouton ratios were restored in the ST rats that were successfully trained to step. Although the frequency and number of synaptic boutons in apposition with $\alpha$-MNs were similar in ST-Non-Tr, ST-Tr, and intact rats, the total number of boutons apposing $\gamma$-MNs was reduced in both ST groups compared with intact rats. In addition, aberrant synaptic contacts were encountered in both experimental groups, as both C- and M-type boutons apposed the soma membrane of $\gamma$-MNs after a neonatal transection regardless of training status, but not in intact rats. Importantly, C-type boutons are of intraspinal origin and may modulate motoneuronal activity during locomotion (Miles et al., 2007). These results are summarized in supplemental Figure 1 (available at www. jneurosci.org as supplemental material), which highlights the changes in bouton type distribution after transection and training.

The ST-induced increase in inhibitory-to-excitatory synaptic input to both $\alpha$ - and $\gamma$-MNs is likely to have reduced the level of recruitment of the MNs. Indeed, previous studies have shown reduced spontaneous integrated electromyographic activity of both the soleus and lateral gastrocnemius muscles in adult cats after a complete spinal cord transection at a neonatal age (Alaimo et al., 1984). From a functional perspective, key findings here were the ST-induced increase in inhibitory influences on both $\alpha$ - and $\gamma$-MNs and that this effect could be overcome in both MN types by step training. Locomotor training maintained the normal relationship between excitatory S-type boutons and inhibitory F-type boutons for both $\alpha$ - and $\gamma$-MNs after ST. The present ultrastructural data are consistent with our recent findings that locomotor training after a neonatal ST increases the amplitude of EPSPs in gastrocnemius MNs while having no significant effect on IPSPs (Petruska et al., 2007).

The potential role of $\gamma$-MNs regulating locomotor behavior after ST and locomotor training was also of special interest for the present study. The excitability of $\alpha$-MNs is normally modulated by $\gamma$-MNs, which control the contractile state of intrafusal muscle fibers within spindles and play a role in the phenomenon of $\alpha$-gamma linkage (Granit, 1975; Taylor et al., 2004). Two functional classes of $\gamma$-MNs have been identified, dynamic and static, and they influence the stretch sensitivity and discharge rate of muscle spindle afferents (Matthews, 1962, 1981). In addition, the discharge of $\gamma$-MNs is regulated by descending pathways from the brainstem and primary afferent activity (Appelberg et al., 1982; Davey and Ellaway, 1989; Baker et al., 1991; Ellaway et al., 1997). Interestingly, an acute SCI results in altered $\gamma$-MN activation patterns. Specifically, $\gamma$-MNs showed regular discharges in an acutely decerebrated cat, whereas an acute thoracic spinal cord transection injury in a decerebrated cat resulted in increased synchrony of $\gamma$-MN discharges for at least the first $24 \mathrm{~h}$ after injury (Davey and Ellaway, 1988). Similarly, the activity of $\gamma$-MNs was not affected by cutaneous afferent stimulation in a decerebrated cat, whereas an acute thoracic spinal cord transection injury in a decerebrated cat resulted in either facilitation or inhibition of $\gamma$-MNs by cutaneous sensory inputs (Ellaway et al., 1997).

Furthermore, studies in clonidine-treated spinal cats suggested that primary afferents fired at an increased mean rate, suggesting a large increase in static $\gamma$-drive, with the elevated rates usually being maintained tonically throughout the step cycle (Bennett et al., 1996). Although the long-term effects of a neonatal SCI on the discharge patterns of $\gamma$-MNs is still unknown, the above studies demonstrated that the physiological properties of $\gamma$-MNs are changed acutely postlesion. In the present study, a markedly impaired locomotor behavior was also demonstrated after the ST, and our ultrastructural data indicate that synaptic plasticity affecting both $\alpha$ - and $\gamma$-MNs may be an important contributor to this impaired locomotor ability. It is conceivable that locomotor training, which normalized the ratio between inhibitory and excitatory inputs to both $\gamma$ - and $\alpha$-MNs, resulted in an overall recovery of excitatory drive to 

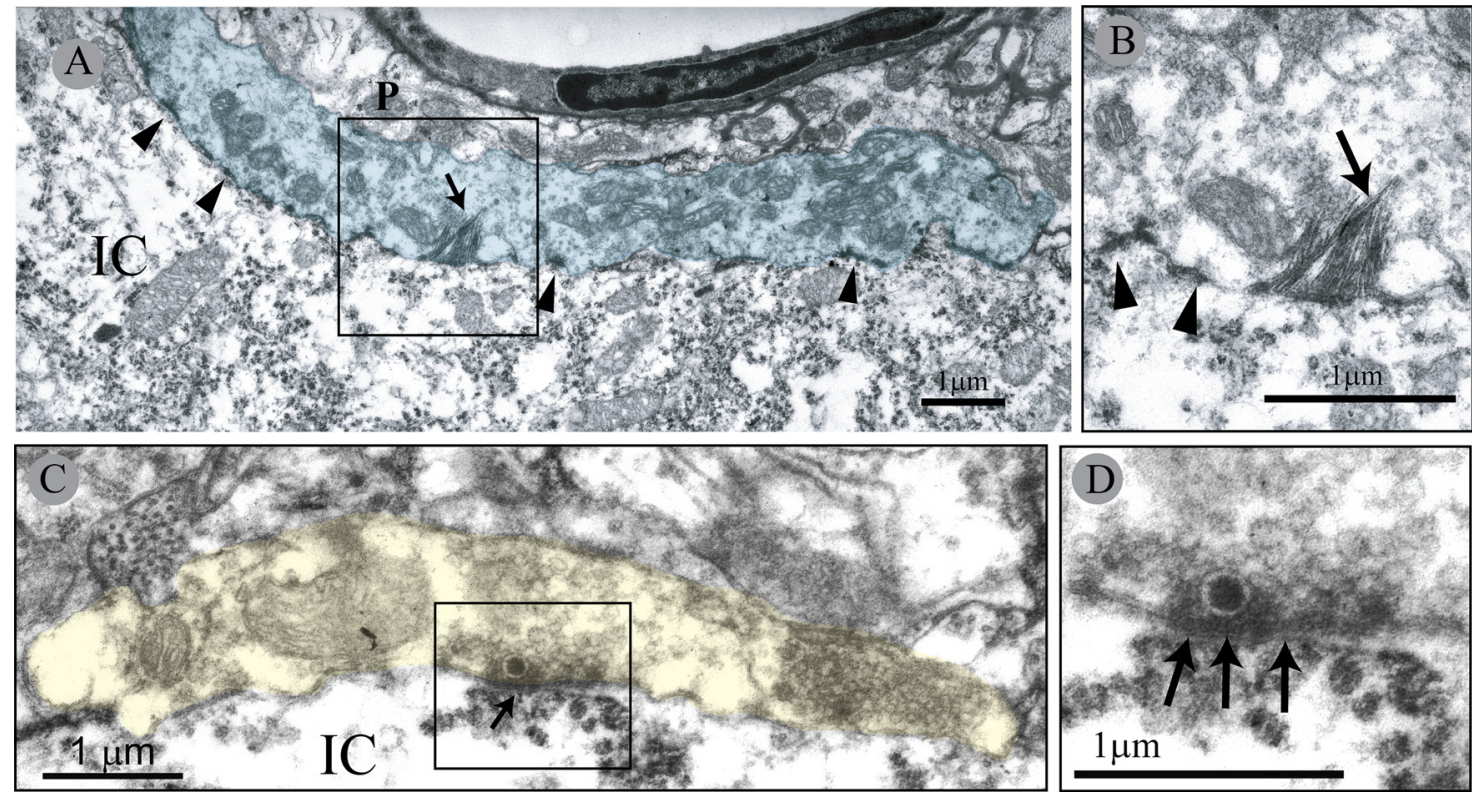

Figure 4. Electron micrographs of M-type bouton apposing a $\gamma$-MN in a neonatally transected rat. $A$, High magnification of the M-bouton (blue). Note multiple active zones (arrowheads) and HRP product crystals (arrow). B, Detail of box in $\boldsymbol{A}$ showing HRP product crystals (arrow) and active sites (arrowheads). C, C-type bouton (yellow) apposing a $\gamma$-MN in a neonatally transected rat. D, Detail of subsynaptic cistern (arrows) associated with the postsynaptic element. Note the presence of a single large dense core vesicle near the presynaptic active zone region. IC, Motoneuron intracellular space; P, P-type bouton.
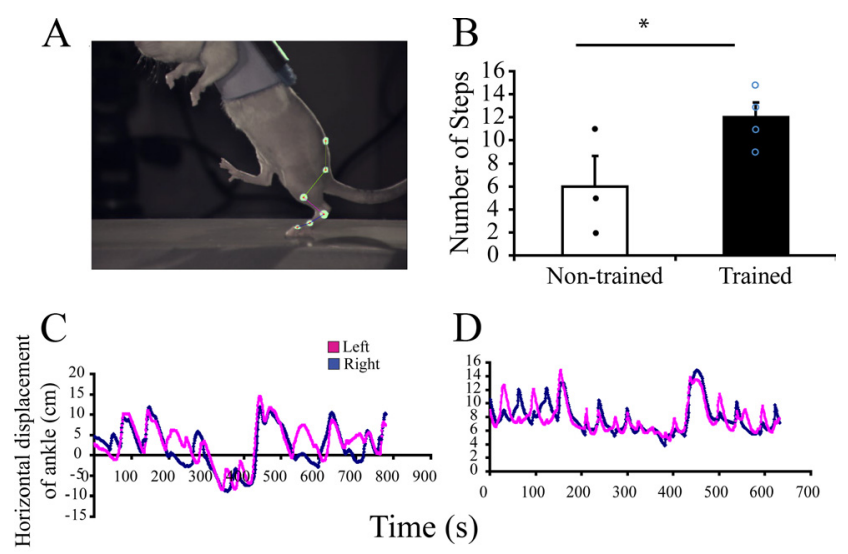

D
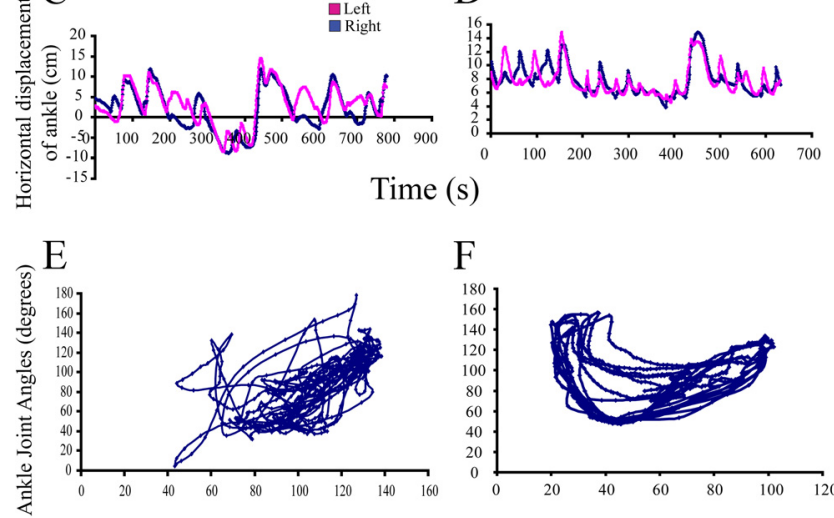

Knee Joint Angles (degrees)

Figure 5. A, Kinematics analysis was performed during bipedal locomotion. Retro-reflective markers placed on bony landmarks were digitized to reconstruct movements. $\boldsymbol{B}$, ST-Tr rats performed a greater number of steps than ST-Non-Tr rats. $\boldsymbol{C}-\boldsymbol{F}$, Analyses from a ST-Non-Tr rat $(\boldsymbol{C}, \boldsymbol{E}$, ) and a ST-Tr rat $(\boldsymbol{D}, \boldsymbol{F})$. Note that the ST-Non-Tr rat performed fewer steps, which were not alternating $(\boldsymbol{C})$ and showed poor intralimb coordination (E). In contrast, the ST-Tr rat performed a greater number of alternating steps $(\boldsymbol{D})$, which were more consistent and better coordinated $(\boldsymbol{F}) .{ }^{*} p<0.05$.

$\gamma$-MNs that, in turn, enhanced $\alpha$-MN excitability through muscle spindle afferents.

Although the relative proportions of synaptic boutons in apposition with MNs differed between groups, the total num- ber and density of synaptic boutons apposing $\alpha$-MNs were similar in intact, ST-Non-Tr, and ST-Tr rats. Previous studies have reported an increased immunoreactivity for the GABAergic marker, GAD67, adjacent to lumbar motoneurons after a thoracic ST in adult cats (Tillakaratne et al., 2002). It is possible, however, that the relative proportions of glycinergic and GABAergic boutons may have changed after ST since F-type boutons in apposition with spinal MNs contain glycine and/or GABA (Örnung et al., 1994, 1996). Quantitative immuno-EM studies will be needed to address this possibility.

An interesting aspect of the present study was the demonstration of prominent plasticity of synaptic input to $\gamma$-MNs after a neonatal ST and locomotor training. We observed a substantially lower number of synaptic contacts apposing $\gamma$-MNs at an adult age after a neonatal ST compared with intact adult rats and this effect was not overcome by locomotor training. This difference was due to significantly lower numbers of both S- and F-type boutons in the transected rats but with a net increase in the F/S ratio. After ST, the decrease in S-type boutons was more prominent than the decrease in F-type boutons in apposition with $\gamma$-MNs. In fact, four of the $15 \gamma$-MNs of ST-Non-Tr rats had no S-type boutons apposing the soma membrane. A similar pattern of a more prominent reduction of excitatory S-type than inhibitory F-type boutons was recently reported to affect spinal $\alpha$-MNs in a rat model of experimental allergic encephalomyelitis, an inflammatory condition affecting intramedullary white matter tracts and causing paralysis (Marques et al., 2006).

A surprising observation was that both $\mathrm{C}$ - and M-type boutons apposed the soma membrane of $\gamma$-MNs in ST rats, regardless of training. Neither of these bouton types contact $\gamma$-MNs in intact rats (Ichiyama et al., 2006) or cats (Lagerbäck et al., 1986; Arvidsson et al., 1987; Destombes et al., 1992; Simon et al., 1996). In fact, the presence of C-boutons is commonly considered a determining criterion for $\alpha-\mathrm{MN}$ identification. Although C-boutons may transiently appose the somatic membrane of $\gamma$-MNs during the early developmental phases in kittens, they are 
completely absent in adult cats (Simon et al., 1996). In the present study, we speculate that neonatal ST may have interfered with this process, and that some C-boutons were never eliminated from the soma membrane of $\gamma$-MNs. C-boutons apposing $\alpha$-MNs also undergo substantial plasticity during development with some C-boutons being phagocytosed by $\alpha$-MNs in kittens as part of the normal early developmental pruning and refinement of synaptic contacts (Ronnevi, 1979). Although the functional significance of C-boutons is not clear, previous reports suggest that they are cholinergic and associated with muscarinic type 2 receptors on motoneuron somata (Hellström et al., 2003). More recent investigations suggest that C-boutons may originate from the medial partition group of interneurons in the spinal cord and regulate motoneuron excitability during locomotion (Miles et al., 2007; Zagoraiou et al., 2009). In the present study, neonatal ST may have interfered with the elimination of C-boutons from the soma membrane of $\gamma$-MNs during development.

The focus on synaptic apposition on the soma, but not the dendrites, of MNs warrants some caution in generalizing these data to the dendritic surfaces. Although somatic synapses are more closely located to the axon hillock, the site for action potential initiation, the vast majority of synapses are distributed over the large dendritic surface of MNs. Following activation of dendritic synapses, the strength of the signal ultimately reaching the $\mathrm{MN}$ soma is influenced by several factors. For instance, the signal transmission from dendrites to the $\mathrm{MN}$ soma depends on both the membrane properties and geometry of the dendrites (Burke et al., 1994; Bui et al., 2003; Rose and Cushing, 2004). In addition, the density of synaptic inputs as well as the F/S ratio may differ between proximal and distal dendritic segments in MNs, thus adding more complexity to the relative contribution of dendritic synapses (Brännström, 1993). For the present studies, the use of HRP as a retrogradely transported tracer was practical for detailed investigations of MN soma synaptology, as the cell bodies were well labeled without the histochemical reaction product obscuring the postsynaptic specializations of the inputs to the soma surface. However, this approach is not compatible with microscopic reconstruction of dendrites over serial sections or ultrastructural analysis of intermediate and distal dendritic structures.

In the present study, rats underwent a complete midthoracic ST at P5 and synaptic inputs to TA MNs were studied in the lumbar spinal cord, primarily within L4. During normal development of descending tracts in rodents, the first corticospinal tract (CST) fibers may enter the cervical spinal cord around the time of birth, the mid- to low-thoracic segments at P3, and the lumbar segments by P4-P7 (Schreyer and Jones, 1982; Gribnau et al., 1986; Schwab and Schnell, 1991). Additional CST fibers enter the spinal cord and progressive myelination of the descending fibers occurs during the next few weeks (Hsu et al., 2006). Thus, it is possible that the neonatal injury prevented, rather than interrupted, supraspinal innervation of the lumbar spinal cord. Comparisons of the present findings with the effects of ST and locomotor training on MN synaptology in adult animal models, however, are needed to understand the locomotor recovery process and associated synaptic plasticity when an SCI interrupts established and fully developed descending and ascending tracts.

The overall fundamental question, however, is whether the observed ultrastructural changes after injury and after locomotor training are large enough to alter the behavior of the motoneurons. Although, $\gamma$-MNs exhibited a large decrease in the innervation density and a large increase in the F/S ratio, the apparent recovery in the $\mathrm{F} / \mathrm{S}$ ratio caused by training was not matched by significant changes in the innervation of either F- or S-type bou- tons. The presence of the large C- and M-type boutons in apposition with $\gamma$-MNs after injury and locomotor treatment is significant, even at modest numbers, as they are expected to be absent for this population of neurons. For $\alpha$-MNs, there also was an injury-induced increase in the F/S ratio. This was corrected with locomotor training by an increase in the innervation by S-type boutons and a decrease in the innervation by F-type boutons, although this was not associated with significant changes in the innervation by F- and S-type boutons.

A limitation in the present data, as in all previous quantitative studies of synaptic inputs onto $\mathrm{MN}$, is that the data are restricted to the somata. Our unusually large selection of retrogradely labeled $\alpha$ and $\gamma$-MN somata for quantitative ultrastructural analysis, however, allowed for a substantial sample size and detailed characterization of the postsynaptic structures. Although the significance of the changes in somatic compared with dendritic innervation to locomotion are not well understood, electrophysiological changes in passive membrane properties (Button et al., 2008) and postsynaptic responses (Petruska et al., 2007) have been recorded from the soma after a complete spinal cord transection. Locomotor training after a neonatal ST injury in rats has been shown to increase the amplitude of EPSPs in gastrocnemius MNs, wheras no effect was noted for IPSP. Yet, we show herein that the innervation by F-type boutons in TA $\alpha$-MNs decrease after training.

Given the multiple components of the spinal locomotor networks that change with training (Edgerton et al., 2004), the ultrastructural changes reported herein may contribute to an improvement in stepping. These changes, however, cannot be assumed to fully explain such a complex behavioral adaptation. Despite the above limitations, we conclude that the ultrastructural signs of synaptic plasticity after a neonatal ST, with and without motor training, appear large enough to have contributed to a change in locomotor behavior. Perhaps the most remarkable aspects of the present results are the degree to which the synaptic properties of MNs can be established and the remarkable level of recovery of locomotor function after the complete elimination of all descending input at such an early stage of development.

\section{References}

Alaimo MA, Smith JL, Roy RR, Edgerton VR (1984) EMG activity of slow and fast ankle extensors following spinal cord transection. J Appl Physiol 56:1608-1613.

Appelberg B, Hulliger M, Johansson H, Sojka P (1982) An intracellular study of rubrospinal and rubro-bulbospinal control of lumbar gammamotoneurones. Acta Physiol Scand 116:377-386.

Arvidsson U, Svedlund J, Lagerbäck PA, Cullheim S (1987) An ultrastructural study of the synaptology of gamma-motoneurones during the postnatal development in the cat. Brain Res 465:303-312.

Baker JR, Catley MC, Davey NJ, Ellaway PH (1991) Influence of the pontine and medullary reticular formation on synchrony of gamma motoneurone discharge in the cat. Exp Brain Res 87:604-614.

Bareyre FM, Kerschensteiner M, Raineteau O, Mettenleiter TC, Weinmann O, Schwab ME (2004) The injured spinal cord spontaneously forms a new intraspinal circuit in adult rats. Nat Neurosci 7:269-277.

Bennett DJ, De Serres SJ, Stein RB (1996) Regulation of soleus muscle spindle sensitivity in decerebrate and spinal cats during postural and locomotor activities. J Physiol 495:835-850.

Brännström T (1993) Quantitative synaptology of functionally different types of cat medial gastrocnemius alpha-motoneurons. J Comp Neurol 330:439-454.

Bui TV, Cushing S, Dewey D, Fyffe RE, Rose PK (2003) Comparison of the morphological and electrotonic properties of Renshaw cells, Ia inhibitory interneurons, and motoneurons in the cat. J Neurophysiol 90:2900-2918.

Burke RE, Fyffe RE, Moschovakis AK (1994) Electrotonic architecture of cat gamma motoneurons. J Neurophysiol 72:2302-2316.

Button DC, Kalmar JM, Gardiner K, Marqueste T, Zhong H, Roy RR, Edgerton VR, Gardiner PF (2008) Does elimination of afferent input 
modify the changes in rat motoneurone properties that occur following chronic spinal cord transection? J Physiol 586:529-544.

Conradi S, Kellerth JO, Berthold CH (1979) Electron microscopic studies of serially sectioned cat spinal alpha-motoneurons. II. A method for the description of architecture and synaptology of the cell body and proximal dendritic segments. J Comp Neurol 184:741-754.

Davey NJ, Ellaway PH (1988) Control from the brainstem of synchrony of discharge between gamma motoneurones in the cat. Exp Brain Res 72:249-263.

Davey NJ, Ellaway PH (1989) Facilitation of individual gamma-motoneurones by the discharge of single slowly adapting type 1 mechanoreceptors in cats. J Physiol 411:97-114.

de Leon RD, Hodgson JA, Roy RR, Edgerton VR (1998) Locomotor capacity attributable to step training versus spontaneous recovery after spinalization in adult cats. J Neurophysiol 79:1329-1340.

Destombes J, Horcholle-Bossavit G, Thiesson D, Jami L (1992) Alpha and gamma motoneurons in the peroneal nuclei of the cat spinal cord: an ultrastructural study. J Comp Neurol 317:79-90.

Dietz V (2009) Body weight supported gait training: from laboratory to clinical setting. Brain Res Bull 78:I-VI.

Edgerton VR, Tillakaratne NJ, Bigbee AJ, de Leon RD, Roy RR (2004) Plasticity of the spinal neural circuitry after injury. Annu Rev Neurosci 27:145-167.

Ellaway PH, Davey NJ, Ljubisavljevic M (1997) Organization of the sural cutaneous input regulating the discharge of triceps surae gammamotoneurones in the cat. Exp Physiol 82:121-138.

Frigon A, Rossignol S (2006) Functional plasticity following spinal cord lesions. Prog Brain Res 157:231-260.

Granit R (1975) The functional role of the muscle spindles: facts and hypotheses. Brain 98:531-556.

Gribnau AA, de Kort EJ, Dederen PJ, Nieuwenhuys R (1986) On the development of the pyramidal tract in the rat. II. An anterograde tracer study of the outgrowth of the corticospinal fibers. Anat Embryol 175:101-110.

Hellström J, Oliveira AL, Meister B, Cullheim S (2003) Large cholinergic nerve terminals on subsets of motoneurons and their relation to muscarinic receptor type 2. J Comp Neurol 460:476-486.

Henry MA, Westrum LE, Johnson LR (1985) Ultrastructure of transganglionic HRP transport in cat trigeminal system. Brain Res 334:255-266.

Hsu JY, Stein SA, Xu XM (2006) Development of the corticospinal tract in the mouse spinal cord: a quantitative ultrastructural analysis. Brain Res 1084:16-27.

Ichiyama RM, Broman J, Edgerton VR, Havton LA (2006) Ultrastructural synaptic features differ between alpha- and gamma-motoneurons innervating the tibialis anterior muscle in the rat. J Comp Neurol 499:306-315.

Kubasak MD, Hedlund E, Roy RR, Carpenter EM, Edgerton VR, Phelps PE (2005) L1 CAM expression is increased surrounding the lesion site in rats with complete spinal cord transection as neonates. Exp Neurol 194: 363-375.

Lagerbäck PA, Cullheim S, Ulfhake B (1986) Electron microscopic observations on the synaptology of cat sciatic gamma-motoneurons after intracellular staining with horseradish peroxidase. Neurosci Lett 70:23-27.

Laporte Y (1979) On the intrafusal distribution of dynamic and static fusimotor axons in cat muscle spindles. Prog Brain Res 50:3-10.

Marques KB, Santos LM, Oliveira AL (2006) Spinal motoneuron synaptic plasticity during the course of an animal model of multiple sclerosis. Eur J Neurosci 24:3053-3062.

Matthews PB (1962) The differentiation of two types of fusimotor fibre by their effects on the dynamic response of muscle spindle primary endings. Q J Exp Physiol Cogn Med Sci 47:324-333.

Matthews PB (1981) Evolving views on the internal operation and functional role of the muscle spindle. J Physiol 320:1-30.

Mesulam MM (1978) Tetramethyl benzidine for horseradish peroxidase neurohistochemistry: a non-carcinogenic blue reaction product with superior sensitivity for visualizing neural afferents and efferents. J Histochem Cytochem 26:106-117.

Mesulam MM, Hegarty E, Barbas H, Carson KA, Gower EC, Knapp AG, Moss MB, Mufson EJ (1980) Additional factors influencing sensitivity in the tetramethyl benzidine method for horseradish peroxidase neurohistochemistry. J Histochem Cytochem 28:1255-1259.

Miles GB, Hartley R, Todd AJ, Brownstone RM (2007) Spinal cholinergic interneurons regulate the excitability of motoneurons during locomotion. Proc Natl Acad Sci U S A 104:2448-2453.

Novikov LN, Novikova LN, Holmberg P, Kellerth J (2000) Exogenous brain-derived neurotrophic factor regulates the synaptic composition of axonally lesioned and normal adult rat motoneurons. Neuroscience 100:171-181.

Olucha F, Martínez-García F, López-García C (1985) A new stabilizing agent for the tetramethyl benzidine (TMB) reaction product in the histochemical detection of horseradish peroxidase (HRP). J Neurosci Methods 13:131-138.

Örnung G, Shupliakov O, Ottersen OP, Storm-Mathisen J, Cullheim S (1994) Immunohistochemical evidence for coexistence of glycine and GABA in nerve terminals on cat spinal motoneurones: an ultrastructural study. Neuroreport 5:889-892.

Örnung G, Shupliakov O, Lindå H, Ottersen OP, Storm-Mathisen J, Ulfhake B, Cullheim S (1996) Qualitative and quantitative analysis of glycineand GABA-immunoreactive nerve terminals on motoneuron cell bodies in the cat spinal cord: a postembedding electron microscopic study. J Comp Neurol 365:413-426.

Petruska JC, Ichiyama RM, Jindrich DL, Crown ED, Tansey KE, Roy RR, Edgerton VR, Mendell LM (2007) Changes in motoneuron properties and synaptic inputs related to step training after spinal cord transection in rats. J Neurosci 27:4460-4471.

Raineteau O, Schwab ME (2001) Plasticity of motor systems after incomplete spinal cord injury. Nat Rev Neurosci 2:263-273.

Ralston HJ3rd (1990) Analysis of neuronal networks: a review of techniques for labeling axonal projections. J Electron Microsc Tech 15:322-331.

Ronnevi LO (1979) Spontaneous phagocytosis of C-type synaptic terminals by spinal alpha-motoneurons in newborn kittens: an electron microscopic study. Brain Res 162:189-199.

Rose PK, Cushing S (2004) Relationship between morphoelectrotonic properties of motoneuron dendrites and their trajectory. J Comp Neurol 473:562-581.

Roy RR, Matsumoto A, Zhong H, Ishihara A, Edgerton VR (2007) Rat alpha- and gamma-motoneuron soma size and succinate dehydrogenase activity are independent of neuromuscular activity level. Muscle Nerve 36:234-241.

Schreyer DJ, Jones EG (1982) Growth and target finding by axons of the corticospinal tract in prenatal and postnatal rats. Neuroscience 7 : 1837-1853.

Schwab ME, Schnell L (1991) Channeling of developing rat corticospinal tract axons by myelin-associated neurite growth inhibitors. J Neurosci 11:709-721.

Simon M, Destombes J, Horcholle-Bossavit G, Thiesson D (1996) Postnatal development of alpha- and gamma-peroneal motoneurons in kittens: an ultrastructural study. Neurosci Res 25:77-89.

Swett JE, Wikholm RP, Blanks RH, Swett AL, Conley LC (1986) Motoneurons of the rat sciatic nerve. Exp Neurol 93:227-252.

Taylor A, Durbaba R, Ellaway PH (2004) Direct and indirect assessment of gamma-motor firing patterns. Can J Physiol Pharmacol 82:793-802.

Tillakaratne NJ, de Leon RD, Hoang TX, Roy RR, Edgerton VR, Tobin AJ (2002) Use-dependent modulation of inhibitory capacity in the feline lumbar spinal cord. J Neurosci 22:3130-3143.

Timoszyk WK, Nessler JA, Acosta C, Roy RR, Edgerton VR, Reinkensmeyer DJ, de Leon R (2005) Hindlimb loading determines stepping quantity and quality following spinal cord transection. Brain Res 1050:180-189.

Ulfhake B (1984) A morphometric study of the soma, first-order dendrites and proximal axon of cat lumbar alpha-motoneurones intracellularly labelled with HRP. Exp Brain Res 56:327-334.

Wolpaw JR, Tennissen AM (2001) Activity-dependent spinal cord plasticity in health and disease. Annu Rev Neurosci 24:807-843.

Zagoraiou L, Akay T, Martin JF, Brownstone RM, Jessell TM, Miles GB (2009) A cluster of cholinergic premotor interneurons modulates mouse locomotor activity. Neuron 64:645-662. 